\title{
Français vs English : histoire d'une rencontre avec penalty... et sans retour
}

\section{Elizabeth Crosnier}

\section{OpenEdition}

\section{Journals}

Édition électronique

URL : http://journals.openedition.org/asp/2973

DOI : $10.4000 /$ asp. 2973

ISSN : 2108-6354

\section{Éditeur}

Groupe d'étude et de recherche en anglais de spécialité

\section{Édition imprimée}

Date de publication : 1 décembre 1997

Pagination : 55-74

ISSN : 1246-8185

\section{Référence électronique}

Elizabeth Crosnier, « Français vs English : histoire d'une rencontre avec penalty... et sans retour », ASp [En ligne], 15-18 | 1997, mis en ligne le 02 mai 2012, consulté le 19 avril 2019. URL : http:// journals.openedition.org/asp/2973 ; DOI : 10.4000/asp.2973

Ce document a été généré automatiquement le 19 avril 2019

Tous droits réservés 


\title{
Français vs English : histoire d'une rencontre avec penalty... et sans retour
}

\author{
Elizabeth Crosnier
}

\section{Introduction}

1 Pourquoi avoir choisi un titre aussi équivoque, et quelque peu frivole, pour un article portant sur la langue de spécialité ? L'allusion explicite aux rencontres de football fera peut-être sourire certains lecteurs, alors que d'autres percevront quelque trace d'amertume ou d'ironie, ou encore verront-ils des propos peu appropriés à la situation. Le fait de poser en parallèle les rencontres bilatérales inhérentes au football et la confrontation francophones/anglophones réduit peut-être la dimension inquiétante du problème et l'austérité du sujet. Lors de notre exposé au GERAS, nous avons introduit une touche d'humour pour traiter d'un sujet a priori sérieux tel que la «chose scientifique » et d'un environnement où, par tradition, le titre révèle tout du traitement et des intentions de l'auteur. Par le biais de dessins humoristiques représentant un joueur de football français essayant désespérément de marquer un but dans le camp anglo-saxon, sachant qu'il n'y aura pas de situation réciproque, nous voulions exprimer la difficulté d'atteindre la cible, de viser juste, en matière de communication en anglais. Toutefois, sur le papier, le ton des propos se fera plus grave. Nous exposerons succinctement les résultats de nos travaux qui seront signalés en fin d'article. Le lecteur trouvera dans nos publications les diverses références bibliographiques.

2 Jusqu'à maintenant, le choix de titres explicites, inspirés du modèle scientifique et approprié au sujet semblait justifié. Le français n'occupe plus de place significative au sein de la communauté scientifique internationale. Une évolution rapide en matière de technologie, et de nouvelles contraintes, ont vu le jour ces dernières années, lesquelles ont pour effet une remise en cause importante notamment de la situation décrite suite à 
nos enquêtes menées en 1990 et 1995 et de nos thèmes d'investigation. De plus, une partie des travaux de recherche en cours semble devoir être interrompue. Par conséquent, nous adopterons ici un angle différent pour exposer quelques réflexions nées de nos recherches et expériences et faire part de nos tâtonnements quant aux nouvelles orientations à prendre dans un contexte aussi mouvant. L'étude menée jusqu'alors de manière rigoureuse laissera la place à un contenu plus subjectif, à des impressions qui ont jailli spontanément et à l'expression plus intuitive. Nous avons voulu rendre compte, très modestement, des points positifs mais aussi des obstacles auxquels nous sommes confrontée en tant que chercheur et enseignante, surtout face à des issues encore floues et un terrain non stabilisé.

\subsection{Contexte}

3 Le français disparaît de la scène internationale, le tout anglophone est imposé par les Native Speakers (NS) et les rencontres sont autant de terrains où s'affrontent, sans espoir de retour, les Non Native Speakers (NNS) pour lesquels les règles du jeu sont claires : Publish in English or Perish. Actuellement, une approche contrastive fondée sur la comparaison de documents authentiques exprimés en français et en anglais perd son sens. La production écrite en français se réduit à une peau de chagrin, quant à la production orale, elle tend à disparaitre des programmes des manifestations ayant lieu en France. Cet article n'a pas pour objectif de déplorer cet état de fait mais plutôt de mettre l'accent sur la nouvelle donne et ses effets. Il vise également à exprimer les hésitations du chercheur en langue de spécialité quant aux choix de ses thèmes de travail et enfin celles de l'enseignant face à un public demandeur. Quel est l'intérêt de chercher des points de repère avec le français sans être décalé dans le temps? Quelle attitude l'enseignant d'anglais de spécialité (ESP) en milieu scientifique français peut-il adopter quand on connaît l'importance de l'observation et de la prise de conscience des différences? Telles sont les questions, entre autres, qu'il faut se poser avant d'amorcer d'autres virages.

Depuis le début de notre recherche sur le sujet en 1989, jusqu'à 1996, nous avons rencontré les membres qui constituent la communauté scientifique afin de connaître leur fonctionnement et leurs productions écrites dans les deux langues. Par conséquent, dans un premier temps, il nous paraît opportun de rappeler quelques résultats particulièrement marquants et surtout nécessaires à la poursuite des travaux. Pour conclure cette première phase, une synthèse et le bilan qui découle des applications menées auprès des étudiants permettront de mettre l'accent sur les points forts, mais aussi les limites et également les espaces non explorés. Ensuite, nous exposerons nos nouvelles perspectives de travail et nous montrerons qu'elles ne peuvent se stabiliser en raison de l'apparition de nouveaux problèmes. En effet, l'implantation de l'anglais entraine des changements de comportements de la part des scientifiques de langue française qui, de ce fait, n'utilisent celle-ci qu'à un niveau de plus en plus local et restreint. Actuellement, les analyses contrastives portant sur des productions récentes s'avèrent impossibles pour la plupart des disciplines, ce qui constitue un obstacle dans la poursuite du travail engagé. Notre objectif qui, dans un premier temps, consistait à envisager une exploration sur la relation entre les productions écrites et orales dans les deux langues, c'est-à-dire entre les articles et les exposés oraux, est nécessairement remis en question. Nous avons alors opté pour une autre trajectoire, c'est-à-dire que nous avons porté notre attention sur des messages exprimés uniquement en langue anglaise et sur les 
incidences de cette option, tout en considérant les perspectives pédagogiques dans le cadre de l'aide à apporter aux scientifiques français en vue de l'utilisation de l'anglais à visée professionnelle.

\subsection{Langue française et communication scientifique internationale}

5 Il est inutile de rappeler les causes et les effets de la disparition du français au profit de l'anglais au sein de la communauté scientifique. La compétitivité, le rôle des lobbies omnipotents qui contrôlent depuis les États-Unis le monde de la recherche et de la publication (SCI, ASME, IEEE), la position de la science française à l'échelle internationale et enfin les restrictions budgétaires pratiquées en France sont quelques-uns des paramètres qui ont contribué au déclin du français et à accélérer le processus. On n'utilise guère le français scientifique; on ne parle plus le néerlandais scientifique depuis longtemps. À titre d'information, 87 publications étaient retenues pour la France par le Science Citation Index (SCI) en 1992 parmi lesquelles 24 étaient rédigées en anglais, 300 l'étaient pour les Pays-Bas, dont une seule était en néerlandais. Un rapide tour d'horizon permet de constater l'état des lieux actuel. Les conséquences sont entre autres :

- la mort des revues françaises de haut niveau rédigées en français. Elles représentent entre 2 et $5 \%$ par rapport aux publications en anglais, notamment dans les sciences exactes et sciences de la terre, pour les revues dites de communication primaire ;

- l'absence presque totale de la langue française dans les rencontres ayant lieu en France. Certains organisateurs utilisent exclusivement l'anglais y compris pour les affiches, les programmes, les informations, les posters et les ateliers ;

- la réduction, voire la suppression, pour des raisons financières et d'organisation, de systèmes de traduction dans les congrès scientifiques. Les deux manifestations auxquelles nous avons assisté en 1996 se sont tenues exclusivement en anglais (cf. la section 3 de cet article) ;

- l'absence de recours à des traducteurs et interprètes suffisamment qualifiés. Les restrictions budgétaires, le manque de moyens, de motivation associés aux problèmes liés à l'évolution de la science et de l'extrême spécialisation des chercheurs en sont les causes essentielles.

De ce fait, si nous portons un regard d'enseignante, comme dernier effet, on notera une inadéquation, dans notre pays, entre la formation en anglais des étudiants en sciences et techniques et les contraintes de la vie professionnelle.

7 Les pouvoirs publics tentent de réagir en instaurant des mesures d'encouragement. Suite à la loi du 4 août 1994, afin de retenir quelques publications, le ministère de la Recherche cofinance des prix et apporte une aide financière pour les articles rédigés en français dans les comptes rendus de l'Académie des Sciences. Il octroie des subventions pour l'organisation de congrès en France ainsi que pour des manifestations scientifiques sur le site du Secrétariat d'État à la recherche, le Carré des Sciences à Paris. Les projets sont acceptés à condition que le français soit utilisé tant pour le contenu que pour tous les documents écrits (affiches, programmes, etc.) ou que des systèmes d'interprétation et de traduction soient mis en place, exception faite pour quelques ateliers excessivement spécialisés.

8 Ce contexte nous incite à exposer quelques-uns des résultats obtenus afin de clarifier notre future démarche. Il est hasardeux de s'aventurer sur des voies instables car les trajectoires sont susceptibles de dévier. Toutefois, quelles que soient les options retenues par le chercheur qui s'intéresse à l'anglais scientifique, les finalités restent constantes 
pour l'enseignant: il s'agit toujours de viser les besoins des individus concernés, de repérer leurs manques et d'adapter au mieux les moyens de combler ces derniers. Tels ont été nos objectifs et nos préoccupations, dont les applications donnent un sens à notre travail. Malgré la remise en cause des orientations précédentes, nous espérons être en mesure de poursuivre ce chemin. La section 2 présente une brève synthèse de notre recherche et de sa mise en oeuvre dans le cadre de l'enseignement.

\section{Synthèse des travaux 1989-1996}

9 Cette section a pour objet de rappeler quelques points importants de notre recherche, les résultats des expérimentations et enfin les premières applications effectuées avec nos apprenants. Nous rappelons que nous avons abordé les textes des articles scientifiques selon plusieurs points de vue et que notre approche s'inscrit dans une démarche plurielle. Nous avons bénéficié de l'apport des diverses orientations connues, telles que, notamment, la sociologie de la science, la linguistique et la rhétorique contrastives, l'analyse informatisée de données textuelles et les théories de la communication.

\section{1. Étude contrastive et notion de genre}

10 Le choix d'une approche contrastive a été dicté par le désir de mettre en lumière les raisons pour lesquelles les messages écrits en anglais par les scientifiques de langue française n'étaient pas toujours perçus correctement par leurs récepteurs. On a pu constater que, sur le plan langagier, le problème de communication des NNS ne semblait pas être lié aux compétences strictement linguistiques. Par conséquent, nous avons supposé qu'en comparant des textes écrits par des NS dans chaque langue, il serait possible de découvrir l'origine de cette incompréhension. C'est ainsi que nous avons pensé à établir et comparer deux ensembles de corpus suffisamment homogènes, constitués de textes authentiques, ciblés et naturellement écrits dans la langue maternelle des auteurs.

11 La notion de genre est de plus en plus familière lorsqu'on s'intéresse à la langue de spécialité et on trouvera ses applications dans la littérature. Nous avons tenté de mettre l'accent sur ses implications et ses limites.

L'article scientifique est défini dans la mesure où il est possible de poser clairement les paramètres qui le spécifient à propos de :

- son utilisation : usage professionnel dans un même contexte de production écrite formelle et hautement spécialisée, dont l'objectif est l'information scientifique et technique, sur des supports de diffusion identiques ;

- caractéristiques professionnelles des usagers : profil scientifique (chercheur, ingénieur et étudiant averti) de l'émetteur et du récepteur dont les facultés d'entendement sur le sujet sont relativement identiques, et qui constitue un public restreint de pairs ;

- caractéristiques socioculturelles des usagers : culture acquise à la fois au sein du groupe social, ainsi que dans le cadre de la formation et du travail (institution, culture de discipline, d'entreprise).

13 Nous rappelons que la situation a été envisagée selon un angle qui positionne les scientifiques français en tant qu'émetteurs de messages exprimés en anglais à destination de récepteurs anglophones, majoritaires dans la communauté internationale. 
Les éléments qui ont été analysés, c'est-à-dire les titres, les abstracts et les introductions, sont intégrés dans cette catégorie en tant que sous-parties s'intégrant dans un genre précis. Les spécificités sont d'autant plus intéressantes dans une étude contrastive qu'elles permettent de découvrir les points communs et les divergences entre les deux parties impliquées. On observe alors que, contrairement aux autres paramètres, les caractéristiques socioculturelles de ces deux groupes offrent des lieux de différences notoires. C'est ainsi que nous avons pu nous orienter dans cette direction en supposant que celles-ci sont à l'origine d'obstacles au niveau de la transmission et du décodage des messages et qu'elles doivent être décelées. Nous avons également exploré les conventions qui y sont inhérentes, les conséquences de leur non-respect et enfin les dangers d'une application généralisée. À partir du moment où des règles de bon usage ont été établies par la communauté de langue anglaise concernant le format, le style, l'organisation du discours, les relations auteur/lecteur, et que cette même communauté a des attentes précises, les manifestations langagières de ces contraintes font l'objet d'un intérêt particulier.

\subsection{Méthodologie de modélisation}

15 L'intérêt de l'approche contrastive réside notamment dans la possibilité de comparer les informations collectées dans chaque corpus, et à propos de chaque groupe, et ainsi de souligner les éléments sujets à variations. La découverte de schémas d'organisation des textes et de stratégies de communication à partir d'une suite d'opérations : lecture de données textuelles informatisées, repérage du visible, sélection d'indices sur la base de fonctions et de notions, et enfin interprétations, ont permis de décrire les processus de communication de manière simplifiée, par la suite modélisables. La construction de modèles a été possible essentiellement grâce à ce repérage consistant.

Les analyses fondées sur le repérage statistique n'ont pas toujours bonne réputation, elles font parfois l'objet de critiques ou elles éveillent la méfiance. Elles sont toutefois fort utiles dans la mesure où elles fournissent des points de départ, qu'elles révèlent ce qui échappe à l'oeil humain dans le cas du "travail artisanal » et enfin elles sont précieuses puisqu'elles contribuent à étayer les interprétations. La comparaison entre les résultats pour chaque langue a démontré que les modèles variaient, qu'ils étaient plus ou moins représentatifs en raison de leur caractère exceptionnel. Les modèles les plus significatifs étaient issus de textes anglais. Ils furent alors retenus pour être par la suite validés par le biais de leur mise en oeuvre. Une première expérience de validation a été menée. Ceci signifie que des textes proposés par des Français en langue anglaise furent entièrement reformulés, c'est-à-dire reconstruits selon le modèle, puis soumis au verdict des anglophones. Le fait que ces derniers reconnaissent la version reconstruite comme étant un discours familier a prouvé la validité de la modélisation.

\subsection{Points de divergences entre les deux communautés}

L'article scientifique est une forme de discours bien spécifique. Les titres, les abstracts et les introductions ont été choisis pour l'analyse en raison du rôle qu'ils jouent et de leur contenu fortement communicatif car pour l'auteur ils assurent la vente de son produit. Parmi les aspects les plus révélateurs de divergences, on peut noter les aspects suivants.

La perception du temps 

thèmes et leur installation, la linéarisation et les déviations par rapport au thème central, la position du connu et du nouveau et la manière de rendre l'ensemble cohérent donnent lieu à des divergences notoires. Le traitement de ces notions trahit l'environnement culturel. Il contribue au confort de lecture et de lisibilité des messages.

\section{L'aspect interactif} celui qui sait et celui qui cherche l'information. Le rôle « dialogal » de l'écrit se traduit à travers la participation sollicitée ou l'autorité, et la manière d'imposer les messages. Le lecteur peut être piloté ou au contraire se trouver en situation d'interprète.

\section{L'argumentation}

20 Parmi les différences ressources, la fonction de référenciation est utilisée afin de manifester les connaissances communes, le consensus, mais elle est aussi au service de l'argumentation au travers des critiques du connu et du choix des cibles pour mieux justifier le bien fondé du nouveau.

\section{L'utilisation de certaines figures de rhétorique}

21 L'art de convaincre, de miser sur le pouvoir effectif des mots, est intégré dans le discours scientifique de façon variable. La pratique du hedging, stratégie à multiples facettes, sert simultanément à couvrir l'auteur en cas de controverse, à exprimer son honnêteté en avouant ses incertitudes et à établir les liens avec ses pairs en répondant à ses attentes en matière de style. En outre, elles s'intègrent logiquement dans la pratique des scientifiques anglo-saxons qui est fondée sur une approche d'argumentation, à la différence des Français qui utilisent plutôt une approche descriptive. Ces stratégies sont d'ordre à la fois diplomatique, économique, scientifique et culturel. Elles sont difficiles à mettre en mots et elles semblent être responsables de difficultés pour les NNS pour lesquels le transfert en anglais, accompagné des nuances nécessaires, correspond rarement, selon Hyland (1996), aux règles de bon usage chez les Anglo-saxons.

Ces trois derniers aspects révèlent l'importance de la relation interactive entre les deux interlocuteurs, la nature de l'échange, la place de l'argumentation et les difficultés langagières qui en résultent pour des auteurs n'appartenant pas aux mêmes communautés de culture.

23 Suite à ce qui vient d'être exposé, on constate effectivement que ces éléments sont étroitement liés à l'environnement socio-économique de chaque groupe. Le mode d'éducation et la culture livresque des étudiants américains, la compétition économique et les liens étroits avec le monde industriel en vigueur aux États-Unis, le sens du collectif influencent le mode d'expression des anglophones et constituent ainsi des d'obstacles que les «étrangers » surmontent difficilement, même en s'imprégnant de leurs modèles. L'usage de la rhétorique est mis au service de la communication scientifique, ce qui la rend plus subjective et va à l'encontre de sa réputation encore actuelle d'objectivité et d'universalité; en France, elle n'est malheureusement pas implantée dans les enseignements au même titre que les éléments purement linguistiques car son maniement est plus délicat. 


\subsection{Avantages au niveau pédagogique}

24

Nous conclurons ce bref aperçu en résumant les points positifs qui permettent de mettre en pratique nos résultats et de concevoir des tâches susceptibles d'aider les étudiants scientifiques de langue française à porter un autre regard sur les faits de communication et peut-être sur le monde anglophone. L'analyse informatisée a fourni des données fiables utilisées comme fondements à nos interprétations. La méthodologie dans le cadre d'une approche contrastive présente l'avantage de développer chez les apprenants la prise de conscience et le rôle actif en matière de :

- connaissance du milieu et de son fonctionnement ;

- connaissance de deux communautés de discours;

- approche de la lecture et du repérage plus appropriée en recherchant ce qui se trouve audelà du visible ;

- nature et manifestations de divergences interculturelles ;

- démarche qui rend l'auteur réellement acteur en développant ses mécanismes à partir des modèles et selon la méthode, à la différence d'une approche prescriptive.

Les scientifiques français, comme tous les NNS utilisateurs de l'anglais à usage professionnel, se voient imposer une double contrainte qui va à l'encontre de situations naturelles : l'action d'utiliser une autre langue et l'action d'adopter une manière d'écrire différente en se référant à des modèles plus ou moins étrangers.

Nous croyons réellement que la possibilité d'expliciter des modèles français, donc familiers, selon une approche inductive, contribue à une meilleure acceptation des divers aspects contraignants, puis facilite l'adhésion. En posant ces points de repère, l'enseignant parvient à atténuer plus aisément les réticences.

\subsection{Exemple d'application}

Voici en quelques mots une application mise en oeuvre dans le cadre de modules de rédaction scientifique. Nous avons sélectionné un abstract écrit par un anglophone sur un problème choisi par nos soins en fonction du public. En premier lieu, nous avons demandé aux étudiants de se positionner en tant que chercheurs et d'écrire en anglais leur propre abstract correspondant à l'article qui traiterait de ce sujet, que nous leur avons exposé brièvement. Ensuite, ceux-ci ont noté les différentes fonctions exprimées dans leur production ainsi que l'organisation générale. À partir de leurs schémas, ils ont repéré leur stratégie de communication. Enfin, ils ont effectué les mêmes opérations sur l' abstract d'origine afin de pouvoir comparer les deux versions. Les discussions qui ont suivi montrèrent qu'ils avaient pris conscience des divergences, qu'ils étaient en mesure de les analyser et de développer leur sens critique pour chacun des textes. En option, nous avons souhaité qu'ils reconstruisent un texte à partir des schémas anglophones, sans toutefois renier l'expression personnelle. Cette proposition a été accueillie favorablement et l'exercice a été bien perçu par tous, il n'a jamais été ressenti de manière négative et les résultats ont été fructueux. L'expérience a été menée auprès d'élèves ingénieurs, de doctorants et enfin d'un groupe de chercheurs confirmés. 


\subsection{Limites}

28 s'appliquent à des textes bien précis, en nombre limité et dans certaines disciplines. Il ne faudrait en aucun cas les généraliser, ce qui signifie qu'un travail identique à propos d'autres sujets mais fondé sur la même méthode serait souhaitable. De même, des études portant sur la discussion/conclusion de l'article pourraient compléter la recherche. Pour les besoins d'une conférence effectuée pour l'école doctorale de chimie, nous avons reproduit notre procédure sur des articles portant sur les procédés industriels; nous avons enregistré des variations au niveau des analyses mais le fonctionnement était réalisable, donc satisfaisant. Ces résultats ne doivent pas être considérés comme universels. En revanche, la méthodologie peut constituer le noyau central de la procédure et nous supposons que les mécanismes peuvent être appliqués à d'autres formes de discours et dans d'autres domaines. Il serait par exemple intéressant d'aborder les sciences de la vie en analysant les rapports médicaux ou même les sciences sociales. Il faut aussi noter que des comportements atypiques, liés à la personne ou à l'institution, annulent les possibilités d'application.

D'autre part, en intégrant des types de textes dans un genre délimité et en extrayant des modèles, il faut être vigilant et éviter l'écueil d'une trop grande normalisation et d'un appauvrissement au niveau de l'expression langagière. À notre avis, cette tendance semblerait incompatible avec la formation des Français. Le danger serait de réduire la communication en calquant uniquement ces modèles et on peut reprocher à cette démarche de nier les initiatives personnelles, les variations et surtout d'aliéner la forme à la fonction du discours. Cette aliénation peut également se percevoir au niveau culturel comme forme de subordination puisque, dans notre cas, la culture anglo-américaine s'impose aux scientifiques français. Nous savons que ces derniers y sont souvent réfractaires et que l'hégémonie de l'anglais, associée à l'image qu'ils se créent des pays concernés, va à l'encontre de leur acceptation des différences. Avec l'expérience de formation de publics scientifiques, nous voulons insister sur ce paramètre non négligeable.

Les Français pourraient percevoir cette rigidité comme un carcan. C'est pourquoi, le formateur en anglais qui intervient auprès de scientifiques déjà impliqués dans l'écriture devra tenter de faire accepter certaines contraintes tout en insistant sur le fait que la réussite de leurs échanges professionnels au niveau international dépend, du point de vue langagier, de leurs compétences autant communicationnelles que linguistiques. Les tâches seront d'autant plus efficaces qu'elles développeront le sens critique et l'action. Car il est aussi souhaitable d'éviter le sentiment d'acculturation qui est susceptible de naître dans notre contexte français. C'est pourquoi nous mettons tant l'accent sur la nécessité de partir de modèles familiers faisant office de repères.

31 Nous avons rappelé certains points positifs et négatifs. Comme nous l'avons déjà signalé, nous avons mis en oeuvre cette méthodologie avec quelques groupes d'étudiants et de chercheurs (2.5). Le travail pédagogique est de longue haleine, mais il semble porter ses fruits. Le problème majeur est lié à l'extrême instabilité du monde scientifique et au fait que nos résultats sont valides maintenant, ce qui nécessite, par conséquent, une réactualisation constante, voire une remise en cause, de la part de l'enseignant. 


\section{Nouvelles orientations : écrit et oral} mis en place en septembre 1995 un projet de création d'un CD-ROM nommé Com-Science, dont l'objectif est d'offrir aux scientifiques français un outil d'aide pour leurs présentations orales dans le cadre de congrès internationaux. Après avoir effectué une enquête auprès des chercheurs dans les diverses disciplines, utilisateurs potentiels, il a été possible de cibler les besoins correspondant à chacune des sections de la présentation et de sélectionner les éléments du discours, en matière de phraséologie, qui permettent de relier les contenus scientifiques. La première étape consistait à répertorier, à partir de documents authentiques regroupés en corpus de phrases, des expressions utilisées par les anglophones et aisément recyclables, selon les fonctions exploitées lors des communications orales. C'est pourquoi nous avons assisté à deux congrès :

- la conférence annuelle du Advanced Summer Institute, ASI'96, Toulouse, du Network of Excellence in Intelligent Control and Integrated Manufacturing Systems (ICIMS-NOE), parrainée par la communauté européenne dans le cadre du programme européen ESPRIT, 74 communications et plusieurs ateliers ;

- la Third International Conference organisée par l'Association Française d'Approximation à Chamonix, 170 communications et des mini symposiums.

Puis, par la suite, il s'agissait de constituer une base de données. C'est cette démarche qui a suscité notre intérêt pour la langue scientifique orale et donné naissance à quelques projets. En outre, la communication orale est relativement peu étudiée par rapport à l'écrit, et elle l'est encore moins dans le contexte scientifique.

semblait a priori intéressante pour la recherche ainsi que du point de vue pédagogique. Nous avons pensé à explorer la relation qui existe entre les deux modes de communication, la publication et la présentation dans le cadre de congrès étant souvent liés. Dans l'impossibilité de transposer vers l'oral les analyses contrastives effectuées précédemment en raison du nombre restreint d'exposés en français, nous avons réorienté notre travail, constitué un corpus uniquement en langue anglaise et choisi la transmission de messages par une même personne via les deux modes : l'écrit et l'oral, avec toutefois des critères identiques qui déterminent le genre de chacune des activités. Par la suite, nous devrons nous interroger sur une autre manière de détecter les sources de difficultés pour les NNS.

Le nouveau thème de recherche n'a pas été choisi au hasard. Il a été dicté à la fois par le désir d'exploiter nos travaux sur l'écrit et les diverses opérations menées dans le cadre du CERDIC, notamment la phase d'enregistrement des présentations des chercheurs anglophones sur les sites de congrès afin de constituer des corpus et collecter ainsi des informations à partir de documents authentiques.

\subsection{Présentation et objectifs}

Dans un premier temps, nous avons abordé la nature de la relation écrit/oral, existante ou non, proche ou lointaine, uni- ou bidirectionnelle, en envisageant les divers cas de figure. En 1996, plus de cinquante présentations effectuées par des anglophones ont été enregistrées et retenues, parmi lesquelles 35 en mathématiques, 8 dans le domaine de 
l'automatisme et des technologies de l'information, et 6 en informatique. Un double problème s'est alors posé : la possibilité d'associer l'écrit et l'oral pour un même auteur, et les conditions d'accès. Pour le premier aspect, plusieurs cas de figure se présentent :

- la présentation est suivie d'un article sur le même sujet : l'oral précède l'écrit officiel. Dans ce cas, l'auteur effectue une préparation orale à partir de ses transparents. Le délai entre celle-ci et la rédaction est très variable, ce qui aura une incidence sur la relation oral/écrit ;

- la présentation est précédée d'un pré-article à la place d'un abstract : l'oral s'intercale entre deux écrits officiels. Les délais sont également variables, mais la présentation est généralement inspirée par le premier écrit ;

- la présentation est suivie d'un article sur un sujet identique mais partiellement ou même complètement modifié : la relation est distante, voire inexistante ;

- l'absence d'article ou de présentation.

Il en résulte un nombre plus réduit d'associations articles/présentations pour un même auteur, par conséquent des corpus de petite taille, puis des modes de relations variables. On pose alors l'hypothèse qu'il puisse y avoir passage d'un état à l'autre, transfert des propriétés de l'un vers l'autre, que les deux sens sont possibles ou que les deux modes de communication sont indépendants. Parmi les problèmes, il faut mentionner le laps de temps qui s'écoule entre le congrès et la disponibilité des articles. Certains auteurs écrivent peu de temps après le congrès. En revanche, d'autres attendront le dernier moment. Se pose également la question de l'accès: autorisation de l'auteur avant la publication officielle, accès par courrier électronique, ou attente des ouvrages et possibilité de les consulter, accès aux congrès, autorisations et conditions matérielles d'enregistrement.

Dans l'état actuel, il nous est impossible de présenter les résultats escomptés puisque les analyses des documents prévues depuis plusieurs mois n'ont pu être réalisées, la majorité des articles n'ayant pas été disponible au moment de l'écriture de cet article. Par conséquent, nous exposerons notre objectif initial, puis nous proposerons seulement quelques remarques globales suite à un travail malheureusement rapide et, enfin, nous soumettrons les questions qui ont surgi spontanément en espérant que les lecteurs ne nous en tiendront pas rigueur. Ceci atteste de la difficulté d'aborder la communication scientifique orale et, notamment, un sujet tel que la relation écrit/oral en raison des conditions matérielles, puis du temps nécessaire au travail d'écoute et de lecture.

Les présentations en congrès durent en moyenne 20 à 30 minutes, dans quelques cas une heure, et sont généralement suivies d'un débat dont la longueur est variable. L'auteur, qui a peu de temps pour exposer l'intégralité de son travail, limite alors sa prestation en donnant la priorité aux faits scientifiques au détriment de la langue générale et de l'aspect conversationnel. Ce dernier se retrouve souvent réduit à quelques expressions simples, standard, peu personnalisées. Pour cette raison et par manque de temps, nous avons délibérément écarté toute idée d'étude nécessitant une comptabilisation des occurrences comme cela avait été effectué pour l'écrit. Le vocabulaire, les marqueurs de cohésion ou les expressions "bouche-trous " propres à l'oral (formules utilisées au quotidien telles que you know, what I want to tell you is..., I'm going to talk about...) ne seront pas analysés malgré leur intérêt et surtout la facilité de repérage. L'objet de ce travail concerne plutôt la relation entre les deux modes de communication en ciblant quelquesuns des aspects déjà mis en lumière par le biais des analyses des articles. La démarche est identique et l'objectif consiste à comparer les stratégies mises en oeuvre dans les communications orales et écrites, les intentions des auteurs et leurs relations avec les 
lecteurs et le public. En référence aux travaux précédents, cette option est apparue comme une suite logique. Malgré le manque de documents et de temps imparti, nous exposons les futures pistes d'investigation.

\subsection{Caractéristiques de l'écrit et de l'oral scientifiques}

40 Nous ne rappellerons pas les définitions de l'expression langagière écrite et orale afin d'éviter les lieux communs. Nous avons déjà vu le rôle dialogal de l'écrit scientifique, propriété qui le rapproche de l'oral. Celui-ci, pour les scientifiques, doit avoir certaines des qualités propres à l'écrit dans la mesure où, pour des raisons de compréhension du contenu scientifique, l'exposé conserve les schémas d'organisation, l'ordre des mots, la pertinence de la langue et la cohérence de l'ensemble. Les lecteurs, comme les auditeurs, ont des attentes communes en ce qui concerne le registre et le style en usage dans la communauté de spécialistes. Le travail de décodage et d'interprétation qu'ils doivent effectuer est similaire sur certains aspects. Il en résulte que les divergences d'ordre culturel repérées au niveau de l'écrit sont susceptibles d'apparaître également à l'oral. On peut supposer que les contraintes en matière de développement du thème et de linéarisation, d'argumentation, de règles de bon usage, les stratégies de communication et les intentions des auteurs se manifestent dans l'expression langagière. En revanche, le présentateur d'un exposé a un champ de liberté plus important au niveau de l'entrée en matière, tout comme l'auteur d'un article dans son introduction.

41 Cette notion de relation entre l'écrit et l'oral mérite d'être encore clarifiée ici. En effet, notons que de nombreux scientifiques utilisent souvent l'expression «faire une communication " pour signifier qu'ils s'expriment oralement devant un public. Ce terme est devenu interchangeable avec celui de présentation, alors qu'il ne l'est pas pour l'article et, de ce fait, on peut croire qu'il se rapproche du mode conversationnel et que leur manière de transmettre des messages varie en fonction du mode. Il est admis que l'information scientifique écrite est véhiculée au moyen de messages impliquant un public de lecteurs potentiels et que l'interaction entre l'auteur et le récepteur est fortement marquée. Nous souhaiterions aborder quelques éléments qui caractérisent cette dernière en observant et comparant les relations de l'auteur de textes avec son lecteur et l'auteur d'exposés avec son auditeur afin de voir dans quelle mesure l'absence ou la présence physique modifie les comportements.

Dans le contexte scientifique, pour mieux comprendre la relation entre l'écrit et l'oral, il faut considérer celle qui s'établit entre le présentateur et son auditoire. L'utilisation massive et généralisée des transparents joue un rôle non négligeable puisque ceux-ci apportent une dimension visuelle fondamentale en se superposant au verbal et en imposant leur contenu indispensable à la compréhension. Par conséquent, l'auditeur se retrouve, en plus, et simultanément, en situation de lecteur de messages verbaux et non verbaux. Dans certains cas, en fonction des sujets ou des présentateurs, il semble qu'un enregistrement pourrait parfaitement se substituer à la présence humaine.

Un autre paramètre joue un rôle important dans cette relation. En raison d'un minutage strict et afin de respecter le contenu des informations, la plupart des scientifiques préparent leur présentation en répétant, souvent à voix haute, à partir de leurs transparents. Certains ont rédigé leur texte auparavant, mais ils privilégient souvent le côté visuel. Par conséquent, l'expression orale tend à perdre une partie de sa spontanéité. Ce fait est d'autant plus marquant chez les locuteurs non anglophones s'exprimant en 
anglais. C'est ainsi que l'oral adopte de plus en plus la forme d'un écrit oralisé. Les parties du discours qui échappent éventuellement à cette règle se réduisent à la mise en condition, à l'introduction si elle existe et à la conclusion si elles existent. Quelques expressions permettent parfois de repérer les différentes sections mais, actuellement, nous ne porterons pas de jugement dans la mesure où notre échantillon n'est pas suffisamment représentatif. La communication orale devient ambiguë, l'oral et l'écrit, le verbal et le non verbal s'épousent, ils deviennent indissociables et surtout chacun est métissé par l'autre.

Ce bref aperçu des paramètres qui jouent un rôle non négligeable dans la construction de messages scientifiques oraux prouve que ces derniers sont à la fois métissés par le visuel et dénaturés par la mémorisation. L'oral non préparé, c'est-à-dire l'improvisation ou l'émission spontanée d'expressions à des fins interactives telles que let me show you, you imagine... dépend de chaque locuteur, ce qui demeure aléatoire et variable, surtout dans le cas des NNS.

\subsection{Remarques}

En raison des circonstances, nous nous limiterons à un repérage rapide et à quelques réflexions survenues spontanément suite à la comparaison des introductions des articles et de l'entrée en matière des exposés. Les centres d'intérêt, comme nous l'avons déjà mentionné, sont identiques à ceux de l'écrit. Ceci signifie que les éléments propres à l'oral, bien que repérés aisément, seront écartés de notre propos, tels que la permutation des pronoms we et I, l'utilisation du pronom you, la forme interrogative des questions de rhétorique, les variations en matière de phraséologie de présentation.

\subsection{Exemples}

Cette section a pour objet de présenter quelques exemples extraits de l'introduction des articles et des exposés des auteurs anglophones afin de repérer les similitudes et les variations entre la version écrite et la version orale. Comme pour l'écrit, les aspects considérés comme lieux de divergences sont l'organisation du discours, l'expression de l'interaction, l'argumentation et l'utilisation de figures de rhétorique (2.3). Un rapide tour d'horizon nous permettra d'orienter les prochaines investigations. Nous rappelons qu'aucun repérage statistique n'a été effectué et qu'il s'agit uniquement d'une ébauche fondée sur des appréciations générales.

\subsubsection{L'organisation du discours}

Il semble que, globalement, l'introduction des exposés tende à suivre un schéma d'organisation proche de celui de l'écrit des anglophones. Dans notre cas, les spécialistes d'automatisme et d'informatique ont suivi le même ordre de présentation du connu et du nouveau et utilisé des fonctions identiques en matière de référenciation et d'argumentation. À première vue, le modèle anglais d'introduction retenu pour l'écrit se retrouverait dans nos corpus, tant à l'écrit qu'à l'oral. Les contextes de congrès étaient spécifiques, l'un étant dans le cadre de projets européens et l'autre portant sur des travaux américains financés par l'armée. Par conséquent, ceci devrait être validé par un échantillon plus vaste et un repérage intensif des divers marqueurs. 
En revanche, il est intéressant de noter que les mathématiciens se différencient par un comportement atypique à l'oral, phénomène qui a déjà été remarqué pour l'écrit. Il faut toutefois être prudent car on peut supposer que la spécificité du contexte soit partiellement responsable de ce phénomène. Par exemple, dans notre cas, les chercheurs appartiennent tous à une association qui leur donne l'occasion de s'exprimer régulièrement dans un cadre assez fermé. La nécessité d'introduire leur travail de manière formelle et conforme à un modèle semble moindre. Effectivement, la plupart entraient rapidement dans la démonstration après quelques mots de mise en condition afin de détendre l'atmosphère et préparer le public à l'austérité du contenu scientifique. Le schéma d'organisation le plus représentatif pourrait se présenter ainsi :

- manière personnelle de mise en condition selon le contexte, l'environnement ;

- a motivation de l'auteur à un niveau individuel ;

- démonstration.

C'est pourquoi les deux versions d'introduction ne sont que rarement calquées, ce qui signifie que peu d'éléments sont comparables. La relation entre les productions d'un même auteur peut offrir tous les cas de figure et tous les traitements possibles. On retrouve, sans grand étonnement, les caractéristiques relevées pour l'écrit, c'est-à-dire cette approche individualisée de la communication. Par conséquent, une étude approfondie nécessiterait des documents de provenances plus variées afin de vérifier ces impressions.

\subsubsection{Argumentation et référenciation}

50 Nos analyses précédentes ont montré les stratégies d'argumentation des auteurs qui exploitaient les diverses facettes de lafonction de référenciation afin de justifier la validité de leurs travaux : la connaissance commune des faits, l'existence de problèmes non résolus, l'insatisfaction des solutions existantes, les références nominatives. Nous avons vu de quelle manière s'établissait le consensus à l'intérieur de la communauté de pairs ; c'est ainsi que se créait l'interaction entre les auteurs et les lecteurs.

Quelques extraits de références par rapport à l'existant et d'expressions de la relation entre le connu et le nouveau permettent de comparer l'écrit et l'oral dans le domaine de l'automatisme et des technologies de l'information et d'orienter le travail d'analyse :

Oral : A complex system will continue to require well-trained personnel, that's not new.

Écrit: Appropriate deployment of human capabilities is now a business critical competence

Oral : Very little has been done on...

When you look at the literature, you will find...

Écrit : aucune remarque comparable

Oral : I'll briefly describe what has happened in this particular area in the last years.

Some people have tried to adopt this methodology...

Écrit: In the last few years the application of Petri Nets has produced interesting results but this is far from achieving a unified modelling...

Oral : But nonetheless the cost is a well-known problem

Écrit : Distributed systems can also suffer from a number of disadvantages such as conflicting sub-process requirements... 


\section{les chercheurs pour justifier l'intérêt qu'ils portent au sujet et annoncer l'objet de leur travail :}

Oral : The key component is that previously manual employees are now expected to use their intellect, and this is a change.

Écrit : One of the main differentiating features of the emerging paradigm is the emphasis on the integration of the intellectual and creative capabilities of manual workers in jobs and skills previously considered purely physical.

Oral : Why have humans become now so important?

Écrit : Business pressures... have given additional significance to the human factor.

Oral : I'd like to make a brief account of why we may need such tools.

Écrit: Given that an efficient process begins with a good design methodology, the development of better design tools is essential. raison des difficultés liées à l'utilisation de documents authentiques et à l'accès à la communauté scientifique, malgré un réel intérêt (associations, congrès, publications ESP). En outre, la mouvance du terrain, la variété des disciplines et les spécificités de tout ordre (culture institutionnelle, contraintes des entreprises, etc.) sont autant d'obstacles qui tendent à freiner, voire entraver les désirs d'exploration, et à décourager les bonnes volontés. Le problème se pose alors de manière cruciale lorsque cette recherche porte sur les applications pédagogiques. En l'absence d'informations significatives, et dans le cas où il est préférable d'éviter l'utilisation de méthodes fondées sur la prescription, un certain nombre de questions se posent à l'enseignant d'anglais en milieu professionnel en France. Nous avons considéré les trois points de vue suivants, valables pour les deux modes de communication, puis nous avons spécifié quelques obstacles propres à l'exposé oral.

\subsection{Contexte psychologique}

56

Quelle attitude le formateur doit-il avoir face à des apprenants NNS dont le degré de réceptivité et d'acceptation de la situation par rapport à l'anglais langue de 
communication internationale est parfois peu élevé? Il faut déjà prendre en considération les résistances de certains scientifiques en activité. Quant aux étudiants, il est nécessaire de les sensibiliser et de leur montrer les lieux où s'appliquent ces différences interculturelles que nous avons découvertes afin qu'ils puissent ensuite entrer dans ce processus de permutation selon la langue utilisée. Il s'agit bien ici d'un mécanisme qui permet de basculer d'un système vers un autre et non pas d'une démarche de subordination. Il existe toutefois des disciplines pour lesquelles cette manoeuvre semble difficile à opérer en raison des différences importantes d'approches entre les communautés anglo-saxonne et française. Les points délicats tels que le traitement typiquement anglo-saxon de cette figure de rhétorique qu'est le hedging nécessite la double maîtrise de la langue et de la culture. La difficulté concernera ensuite l'enseignement de cette technique.

\subsection{Aspect éducatif et culturel}

57 De quels points de repère l'enseignant disposera-t-il en l'absence de sources d'informations en français, connaissant l'importance de l'observation et de la prise de conscience des divergences entre les deux communautés comme étapes préalables au passage à l'acte d'intégration et d'imitation? L'utilisation de documents moins récents semble peu appropriée dans des contextes à évolution rapide et lorsqu'il est nécessaire de respecter les règles en vigueur au sein de la communauté. D'autre part, à la différence des États-Unis, la France ne diffuse pas un enseignement scientifique dans lequel la connaissance livresque et le rôle des références exploitables au niveau de l'argumentation occupent une part prépondérante. Il en est de même pour l'usage de la rhétorique au service de l'information scientifique. Par conséquent, ces différences de formation constituent des obstacles difficiles à gérer.

\subsection{Point de vue linguistique}

58 Les points délicats qui viennent d'être considérés sont rarement abordés par les enseignants dans le cadre de la formation en langue scientifique car difficiles à appréhender en raison de la subtilité et des nuances qui leur sont inhérentes. Nous pensons que :

59 - ils doivent être insérés dans un discours global et une formation générale en collaboration avec les scientifiques. Ils ne peuvent pas être fragmentés ni extraits du contexte ;

60 - ils nécessitent une double attention impliquant une pratique de la langue, et celle des règles consensuelles et des traditions dans la culture cible ;

61 - ils requièrent une aptitude à mettre en éveil les ressources langagières et à les adapter à un contexte spécifique à un moment donné par le biais de l'improvisation.

62 Ces besoins ont été clairement exprimés par les chercheurs interrogés préalablement par l'équipe du CERDIC. Les pourcentages obtenus font apparaître une demande d'aide pour améliorer les performances notamment en matière de techniques de prise de parole. 


\subsection{Food for Thought}

63 Il est souhaitable que, comme le texte, la prestation orale en anglais des NNS corresponde au mieux aux attentes des anglophones. Nous poserons le problème de l'acceptabilité de l'exposé en termes différents par rapport à l'écrit. En ce qui concerne celui-ci, les attentes sont celles des referees des comités de lecture exigeants en matière de précision. Mais les productions écrites par les NS s'offrent comme modèles et provoquent le phénomène d'imitation spontanée qui, nous l'avons déjà constaté grâce à notre expérience de traductrice, entraîne des résultats souvent positifs au niveau de la lisibilité, laquelle peut être améliorée par le travail de préparation. En revanche, pour l'exposé oral, les messages sont tellement furtifs que ce phénomène ne fonctionne pas aussi aisément. De plus, le locuteur n'est pas totalement épargné de cette part d'improvisation en dépit de la préparation a priori de sa présentation orale. Il semble important, dans le cadre de cette réflexion, de rappeler le rôle de l'affectivité dans l'usage d'une langue autre. Même si ces phénomènes sont connus, ils sont souvent négligés dans l'enseignement des langues. Comme nous l'avons déjà mentionné (2.4), cette situation n'est pas naturelle et la double contrainte qu'elle implique est encore plus forte pour l'oral car elle est accrue par la charge émotionnelle qui résulte de la présence physique et du contexte. Parmi les scientifiques interrogés, beaucoup ressentent cette pression qui inhibe les facultés d'expression en langue qui devient de plus en plus "étrangère "; ils considèrent d'ailleurs que ce facteur dissuade les plus hésitants de faire des exposés dans ces conditions, ce qui est regrettable. Le manque d'aisance dans la langue étrangère et d'assurance face à l'auditoire anglophone les pénalise en les contraignant à s'exprimer de manière plus directe et réductrice de la pensée. Nous devons nous interroger sur les manières de préparer au mieux ces candidats soumis à l'épreuve de l'oral qui signifie, outre l'acceptation du statut de l'anglais comme pour l'écrit :

- - vaincre l'angoisse liée à la présence d'un auditoire anglophone ;

- - accepter la contrainte pour gérer au mieux l'aspect linguistique ;

- - revoir la représentation qu'ils ont parfois des pays anglo-saxons à travers les auditeurs anglophones.

En l'absence d'outils de comparaison et des repères familiers fournis par les modèles français dont nous parlions pour l'écrit (2.4), la possibilité d'imiter les anglophones en réutilisant des éléments fournis grâce au matériau authentique enregistré en anglais dans le cadre professionnel présente un intérêt incontestable. C'est l'option qui a été retenue par notre équipe du CERDIC pour la conception du CD-ROM Com-Science, suite aux constatations des chercheurs interrogés au préalable. Notre objectif de formateur reste inchangé. Comme pour l'écrit, il nous paraît indispensable de fournir aux utilisateurs de l'anglais scientifique un matériau oral vrai, avec sa multiplicité et son instabilité, dans des contextes professionnels réels et, en outre, de les familiariser à la prise de parole en public en anglais professionnel.

Compte tenu des obstacles et du contexte, on réalise que, par rapport à l'écrit, la formation à l'oral scientifique anglais pose des problèmes spécifiques. Nous allons terminer cette section en attirant l'attention sur deux phénomènes qui nous préoccupent. Il faut signaler que l'effet de la prestation scientifique orale d'un NNS en anglais sur l'auditoire est moindre car le seuil de tolérance des anglophones est généralement plus bas dans un contexte international. Il serait alors prudent de s'interroger sur la 
conséquence, c'est-à-dire notamment dans quelle mesure cet anglais métissé et plus ou moins réduit par le filtre de l'émotivité est acceptable. Ensuite, le risque d'acculturation mérite d'être mentionné car l'absence du français comme point de référence d'une part, et objet de comparaison de l'autre, freine chez certains utilisateurs le phénomène d'imitation. Cet aspect psychologique ne peut pas être totalement exclu de la démarche pédagogique. Nous avons tendance à croire, d'après nos expériences et nos entretiens, que le formateur d'expression orale devra s'en préoccuper en premier lieu.

En annexe de cet article, nous aimerions faire part de quelques réflexions sur un domaine peu exploré mais non dénué d'intérêt par rapport à la communication scientifique orale qui nous concerne, à savoir l'essor actuel de la vulgarisation scientifique de haut niveau, en référence aux travaux des Français Jacobi, Mortureux et al. La participation de personnalités éminentes et reconnues dans les contextes les plus spécialisés, s'adressant dans ce cas à un public plus large et essentiellement français, peut contribuer à maintenir la langue française comme véhicule de la pensée scientifique en France et redynamiser la diffusion de l'information, quel qu'en soit le support. Grâce aux moyens technologiques actuels tels que la muséographie avec l'utilisation de ressources multimédia, de bornes interactives où les visiteurs deviennent acteurs, la transmission des informations émises à un public de non-spécialistes pourrait jouer un rôle de détonateur. Il n'est pas de notre propos d'entrer ici dans la polémique de la défense de la francophonie mais plutôt d'imaginer un effet boomerang sur la communauté scientifique en réinstaurant une situation plus propice à l'utilisation du français scientifique, au multilinguisme et enfin à un rééquilibre de la position de l'anglais. Par exemple, dans la mesure du possible, les exposés des NNS pourraient être effectués dans la langue maternelle ou une langue autre au choix des intervenants, en fonction des origines et compétences du public. Les débats pourraient fonctionner de manière identique. Ceci tendrait à réduire l'effet inhibiteur ainsi que les frustrations engendrées par un usage contraint d'une langue mal maîtrisée et représentative d'une culture peu familière. Nous ignorons si de telles expériences ont déjà été menées dans le monde. Ce système, utilisé parfois pour l'oral conversationnel ou dans les contextes informels des congrès, a déjà fait ces preuves. Nous serions tentée de croire que la présence d'intervenants français dans les rencontres internationales serait améliorée tant du point de vue quantitatif que qualitatif. Les enseignants d'expression française et d'anglais pourraient participer activement à la préparation afin d'assurer la recevabilité des messages. Ne serait-ce pas là un remède envisageable à notre problème de rencontres «sans retour " ? Pour conclure notre exposé lors du congrès du GERAS 1997, nous avons anticipé en montrant un dessin humoristique représentant un joueur de football français qui parvenait, enfin, à marquer un but dans le camp anglo-saxon adverse. La cible était atteinte, le message était parvenu à destination.

Je tiens à remercier les membres de l'équipe du CERDIC qui travaille sur le projet Com-Science :J.P. Soula, A. Hernandez et surtout $H$. Dauvergne et B. Crosnier qui ont apporté leur contribution par le biais de remarques pertinentes et de conseils judicieux. 


\section{BIBLIOGRAPHIE}

Brown, G. \& G. Yule. 1983. Discourse Analysis. Cambridge : Cambridge University Press.

Connor, U. 1996. Contrastive Rhetoric. Cross-Cultural Aspects of Second Language Writing. Cambridge : Cambridge University Press.

Crosnier, E. 1990. «L'anglais de spécialité en France : étude des problèmes et besoins des scientifiques et techniciens ». Mémoire de DEA préparé sous la direction de J-P. Soula, Université de Toulouse le Mirail.

Crosnier, E. 1995. «Les scientifiques français et la publication en langue anglaise : contraintes, obstacles et perspectives de solutions d'aide à la rédaction. Étude contrastive de corpus anglaisfrançais informatisés d'informatique, de mathématiques et de mécanique (titres, abstracts et introductions) ». Thèse de doctorat préparée sous la direction de J.-P. Soula, Université de Toulouse le Mirail.

Crosnier, E. 1996. «L'intérêt de la modélisation comme aide à la rédaction en anglais pour les scientifiques français ». ASp 11-14, 89-102.

Crosnier, E. 1997. «Étude contrastive de modèles établis à partir de textes scientifiques en français et en anglais en vue de l'apprentissage de l'anglais L2 ». Cahiers de Lairdil 4-8Questions d'articles. L'article scientifique ,14-29.

Hyland, K. 1996. « Hedging your bets in academic discourse ». Insights IATEFL 1, 39-44.

Martin J. 1996. « Les enjeux du discours scientifique ». ASp 11-14, 13-31.

Salager-Meyer, F. 1995. «I think that perhaps you should: A study of hedges in written scientific discourse in functional approaches to written text. Classroom applications ». The Journal of TESOL - France 1, 127-143.

\section{RÉSUMÉS}

Derrière ce titre énigmatique se dissimule une réalité peu amusante pour la plupart des scientifiques français touchés par le syndrome du «Publish or Perish». Dans cet article, nous rappellerons tout d'abord la prépondérance de l'anglais au sein de la communauté de chercheurs. Puis nous dresserons le bilan de nos travaux sur le français et l'anglais scientifiques. À ce stade de notre étude contrastive sur l'écrit, nous ressentons la nécessité de présenter une synthèse et de rappeler quelques points essentiels. Enfin nous exposerons nos nouvelles orientations puisque, de nos jours, les productions en français sortent rarement de l'antre de leurs auteurs, qu'il s'agisse des publications ou des présentations orales. Ce phénomène nous conduit à en examiner les retombées, puis à explorer un aspect différent, notamment les relations entre l'écrit et l'oral à travers les productions des scientifiques anglophones.

This enigmatic title conceals the unenjoyable reality of most French scientists concerned with the "Publish or Perish" syndrome. Firstly we recall that the English language is largely implanted within the researchers' community. Then we give a review of our research work on scientific French and English. Now we have reached a stage when a synthesis of our contrastive studies on writing should be attempted and major findings highlighted. Finally, we consider new 
orientations since nowadays both written and oral productions in French very rarely reach beyond their authors' working environments. Thus we examine the consequences and start investigating a different field, notably the relation between writing and oral through Englishspeaking scientists' productions

INDEX

Mots-clés : communauté scientifique, enseignement de l'anglais de spécialité, étude contrastive, production scientifique écrite, production scientifique orale, stratégie de communication

Keywords : communication strategy, contrastive analysis, ESP teaching, oral scientific production, scientific community, written scientific production

\section{AUTEUR}

\section{ELIZABETH CROSNIER}

